\title{
AN ANISOTROPIC-WET-ETCHED PITCH OR ROLL MODE-MATCHED GYROSCOPE WITH SLANTED QUADRATURE-CANCELLATION ELECTRODES
}

Haoran Wen, Samuel Wisher, and Farrokh Ayazi

Georgia Institute of Technology, Atlanta, Georgia, USA

\section{ABSTRACT}

This paper presents the design, fabrication, and preliminary characterization of a novel anisotropic-wet-etched silicon pitch or roll gyroscope fabricated on a silicon-on-insulator (SOI) wafer without using the deep reactive ion etching (DRIE) process. The novel gyroscope features slanted electrodes with sub-micron gaps, which for the first time, enables electrostatic quadrature tuning in out-of-plane gyroscopes. Complete quadrature cancellation and perfect mode-matching of the drive and sense modes at $200 \mathrm{kHz}$ are demonstrated in fabricated devices. Sensitivity characterization measures a scale factor of $35.4 \mathrm{pA} / \% / \mathrm{s}$ under quadrature-cancelled mode-matched condition, showing $\sim 10 \times$ improvement in scale factor compared to operation without quadrature compensation.

\section{INTRODUCTION}

MEMS gyroscopes offer self-contained rotation measurement of an object. The most critical application of MEMS gyroscope is to enable miniaturized inertial navigation systems (INS) for personal navigation [1-3]. Personal INS requires tri-axial rotation sensing with high-performance gyroscopes. Different types of MEMS yaw gyroscopes have demonstrated promising performance [4-6]. However, developing high-performance out-of-plane pitch and roll gyroscopes is known to be very challenging.

The performance of a gyroscope can be evaluated through its signal to noise ratio (SNR). The SNR of a gyroscope can be increased by noise reduction and improvement in rate sensitivity. The best way to achieve large sensitivity is through mode-matched operation, with the drive and sense modes having the exact same resonance frequency. When mode-matched, Coriolis force excites the sense mode at its resonance frequency, leading to a $Q$ amplified sense response. However, perfect mode-matching may not be possible due to cross-coupling of resonance modes (i.e. quadrature error) caused by fabrication non-idealities. Quadrature error breaks the eigenvalue-degeneracy of the equations of motion, resulting in a veering phenomenon [7], which appears as a minimum obtainable frequency-split between the drive and sense modes of a gyroscope. In addition, quadrature error provides a path through which drive-loop noise is carried to the sense mode and becomes a major noise contributor in sense output signal.

Considering both effects, quadrature error significantly degrades the output SNR and must be minimized to achieve best performance in a MEMS gyroscope. This is generally done by electrostatic quadrature tuning in mode-matched yaw gyroscopes [8]. However, in out-of-plane gyroscopes that are used for pitch and roll detection and fabricated using wafer level processing and DRIE techniques, mode alignment or quadrature tuning electrodes are typically unavailable, which makes quadrature error the biggest obstacle in realizing high-performance pitch and roll gyroscopes.

In this paper, we show that slanted electrodes along wetetched (111) surfaces can be used for efficient quadrature nulling in out-of-plane pitch and roll gyroscopes. In addition, we extend the use of anisotropic-wet-etching to the fabrication of the entire vibrating body. By doing so, we not only make the frequency split between drive and sense modes insensitive to thickness variations of the SOI device layer, but also eliminate the use of expensive DRIE in device fabrication, reducing both process variation dependency and fabrication cost of the gyroscope.

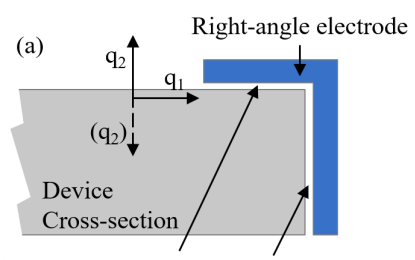

$g_{h}=g_{0} \pm q_{2} ; g_{v}=g_{0}-q_{1}$

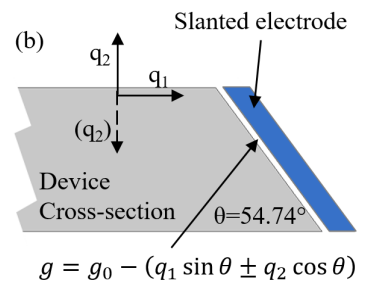

Figure 1: Electrodes in out-of-plane gyroscopes. (a) Conventional horizontal and vertical electrode and (b) novel slanted electrode.

\section{DESIGN AND SIMULATION}

Slanted electrode for quadrature tuning

A general MEMS gyroscope with stiffness non-ideality can be represented by a two-degree-of-freedom spring-mass-damper system with the equations of motion described by:

$m\left[\begin{array}{c}\ddot{q}_{1} \\ \ddot{q}_{2}\end{array}\right]+\left[\begin{array}{c}\dot{q}_{1} \\ \dot{q}_{2}\end{array}\right]\left[\begin{array}{cc}b_{11} & 2 \lambda m \Omega \\ -2 \lambda m \Omega & b_{22}\end{array}\right]+\left[\begin{array}{l}q_{1} \\ q_{2}\end{array}\right]\left[\begin{array}{ll}k_{11} & k_{12} \\ k_{21} & k_{22}\end{array}\right]=\left[\begin{array}{c}F_{d} \\ 0\end{array}\right],(1)$ where $q_{1}, q_{2}, k_{11}, k_{22}$ are displacements and effective stiffness of the drive and sense modes, respectively; $k_{12}$ and $k_{21}$ are crosscoupling spring constants, causing quadrature errors. The eigenfrequency of Eqn. (1) is given by:

$$
\omega_{1,2}=\sqrt{\frac{1}{2 m}\left(k_{11}+k_{22}+2 k_{12} \pm \sqrt{\left(k_{11}-k_{22}\right)^{2}+4 k_{12}^{2}}\right)}
$$

For an ideal mode-matched gyroscope, the stiffness matrix $\boldsymbol{K}_{m}$ is a scalar matrix with $k_{11}=k_{22}=k$ and $k_{12}=k_{21}=0$, which gives degenerate eigen-frequencies $\omega_{1}=\omega_{2}=(k / m)^{1 / 2}$. In practice, $k_{11} \neq k_{22}$ and $k_{12}=k_{21} \neq 0$, which results in divergence of frequencies. To compensate for this non-ideality, electrostatic tuning is usually used. In capacitive transduction, electrical energy stored in the capacitor between vibrating structure and a fixed electrode is a function of $q_{1}$ and/or $q_{2}$, which gives rise to an electrostatic stiffness matrix:

$$
\boldsymbol{K}_{e}=-\left[\begin{array}{cc}
\frac{\partial^{2} U_{e}}{\partial q_{1}{ }^{2}} & \frac{\partial^{2} U_{e}}{\partial q_{1} \partial q_{2}} \\
\frac{\partial^{2} U_{e}}{\partial q_{1} \partial q_{2}} & \frac{\partial^{2} U_{e}}{\partial q_{2}{ }^{2}}
\end{array}\right]=\frac{-V^{2}}{2}\left[\begin{array}{ll}
\frac{\partial^{2} C\left(q_{1}, q_{2}\right)}{\partial q_{1}{ }^{2}} & \frac{\partial^{2} C\left(q_{1}, q_{2}\right)}{\partial q_{1} \partial q_{2}} \\
\frac{\partial^{2} C\left(q_{1}, q_{2}\right)}{\partial q_{1} \partial q_{2}} & \frac{\partial^{2} C\left(q_{1}, q_{2}\right)}{\partial q_{2}{ }^{2}}
\end{array}\right]
$$

The overall stiffness of a gyroscope is the superposition of $\boldsymbol{K}_{m}$ and $\boldsymbol{K}_{e}$ from all electrodes. By changing tuning voltages at different electrodes, the overall stiffness can be adjusted. However, electrostatic tuning in pitch and roll gyroscopes using in-plane and out-of-plane modes has limited efficiency. Conventional gyroscopes fabricated with directional etching allow only vertical and horizontal electrodes (Fig. 1a). Vertical capacitance has negligible change under out-of-plane displacements and horizontal capacitance has negligible change under in-plane displacements. Consequently, any electrode combination gives a capacitance as an uncorrelated function of in-plane and out-of-plane displacements, namely, $C\left(q_{1}, q_{2}\right) \approx C_{1}\left(q_{1}\right)+C_{2}\left(q_{2}\right)$. For example, in a right-angle electrode, the electrical energy neglecting the small contribution from fringing field is given by:

$$
U_{e} \approx \frac{V^{2}}{2}\left(C_{\text {vertical }}+C_{\text {horizontal }}\right)=\frac{\varepsilon_{0} V^{2}}{2}\left(\frac{A_{h}}{g_{h}}+\frac{A_{v}}{g_{v}}\right)
$$


In order to achieve efficient tuning, small gap sizes and large transduction areas are generally used for the electrodes. Compared to the gap size, the area has negligible change under resonant deformations. Assume without the loss of generality that both horizontal and vertical capacitors have same rest gap size $g_{0}$. A series expansion of the electrical energy gives:

$$
\begin{aligned}
& U_{e}=\frac{\varepsilon_{0} V^{2}}{2}\left(\frac{A_{h}}{g_{0}-q_{1}}+\frac{A_{v}}{g_{0} \mp q_{2}}\right) \\
& \approx \frac{\varepsilon_{0} V^{2}}{2 g_{0}}\left[A_{h}+A_{v}+A_{h} \frac{q_{1}}{g_{0}} \pm A_{v} \frac{q_{2}}{g_{0}}+A_{h}\left(\frac{q_{1}}{g_{0}}\right)^{2}+A_{v}\left(\frac{q_{2}}{g_{0}}\right)^{2}+\cdots\right]
\end{aligned}
$$

According to (3), the corresponding $\boldsymbol{K}_{e}$ is diagonal:

$$
\boldsymbol{K}_{e}=\left[\begin{array}{ll}
k_{e 11} & k_{e 12} \\
k_{e 21} & k_{e 22}
\end{array}\right]=\frac{-\varepsilon_{0} V^{2}}{g_{0}{ }^{3}}\left[\begin{array}{cc}
A_{h} & 0 \\
0 & A_{v}
\end{array}\right],
$$

which means in-plane or out-of-plane frequency can be tuned independently, but no quadrature tuning is achieved.

In contrast, anisotropic wet etching of (100) SCS forms slanted sidewalls bounded by (111) crystal planes, which offer a well-defined correlation between in-plane and out-of-plane degrees of freedom. Slanted electrodes along wet-etched (111) surfaces have capacitive gaps affected by both in-plane and out-of-plane motions (Fig. 1b). The energy stored in a slanted electrode is:

$$
\begin{aligned}
& U_{e}=\frac{\varepsilon_{0} A V^{2}}{2 g_{s}}=\frac{\varepsilon_{0} A V^{2}}{2\left[g_{0}-\left(q_{1} \sin \theta \pm q_{2} \cos \theta\right)\right]} \\
& \approx \frac{\varepsilon_{0} A V^{2}}{2 g_{0}}\left[1+\frac{q_{1} \sin \theta \pm q_{2} \cos \theta}{g_{0}}+\left(\frac{q_{1} \sin \theta \pm q_{2} \cos \theta}{g_{0}}\right)^{2}+\cdots\right]
\end{aligned}
$$

Correspondingly, the electrostatic stiffness matrix $\boldsymbol{K}_{e}$ is:

$$
\boldsymbol{K}_{e}=\left[\begin{array}{ll}
k_{e 11} & k_{e 12} \\
k_{e 21} & k_{e 22}
\end{array}\right]=\frac{-\varepsilon_{0} A V^{2}}{g_{0}{ }^{3}}\left[\begin{array}{cc}
\sin ^{2} \theta & \pm \sin \theta \cos \theta \\
\pm \sin \theta \cos \theta & \cos ^{2} \theta
\end{array}\right]
$$

As shown in (8), the electrostatic stiffness matrix has non-zero offdiagonal terms that are suitable for quadrature cancellation. In addition, anisotropic-wet-etching is a self-bounded process. It provides precise control of the surface finish and final geometry, which is critical for gyroscope fabrication.

\section{Gyroscope design and simulation}

A novel single-crystal silicon (SCS) pitch or roll gyroscope with slanted quadrature electrode is designed, in which the entire vibrating structure is formed by anisotropic-wet-etching of SCS.

The anisotropic-wet-etched gyroscope features an isosceles trapezoid cross-section (Fig. 2). The top width of the trapezoid is defined by lithography, whereas the bottom width is determined by both the top width and the thickness of the device, which introduces thickness dependency to the in-plane resonant frequency of the gyroscope. As a result, the in-plane and out-ofplane modes track each other over device thickness variations, making the frequency split thickness insensitive.

Figure 2 shows the electrode configuration and mode shapes of the gyroscope. Horizontal and slanted electrodes with submicron gap sizes are used for capacitive transduction. A pair of slanted electrodes is used to actuate the in-plane drive mode at 200 $\mathrm{kHz}$ while excitation of out-of-plane sense mode is avoided due to opposite mode symmetry at the electrodes. Another two slanted electrodes are used for drive mode current output and electrostatic quadrature tuning. Horizontal electrodes on top of the device are used for differential sense output and sense mode frequency tuning. The sense mode is designed to have a slightly higher frequency than drive mode to ensure mode-matching capability.
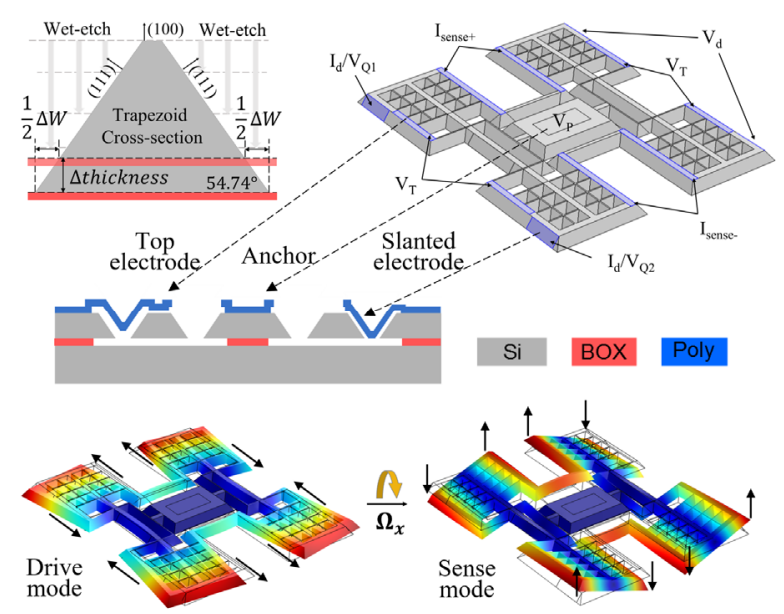

Figure 2: Anisotropic-wet-etched gyroscope geometry and mode shapes. Cross-section shows the shape of the gyroscope body and poly electrode at different locations. The width of the trapezoid structure is coupled to its thickness through the slanting angle.
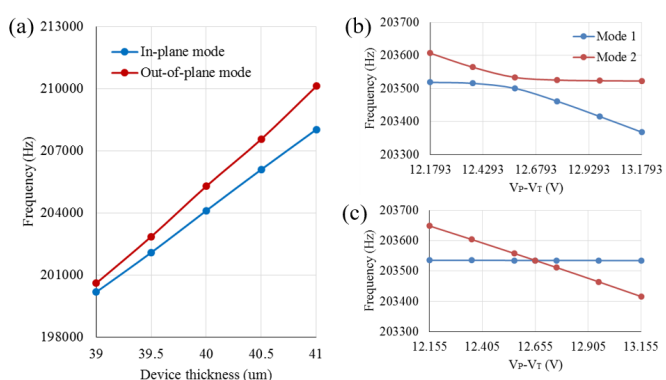

Figure 3: FEM simulation results: (a) frequency variation with different device thickness; (b), (c) frequency tuning w/o and with electrostatic quadrature cancellation.

The frequency behavior of the gyroscope is simulated using COMSOL Multiphysics. Figure 3a shows the thickness dependency of the operation modes. Across $2 \mu \mathrm{m}$ thickness variation, the resonance frequencies change significantly, whereas the frequency difference remains relatively unchanged. The modematching behavior is shown in Fig. $3 b$ and $3 c$. Small trench tilting is added intentionally to simulate imperfections causing quadrature. In simulations, the sense mode frequency is tuned down by changing tuning voltage $V_{T}$ to reduce frequency-split. As shown in Fig. 3b, in the presence of quadrature, frequency veering phenomenon is seen and the two eigen-modes cannot be matched. In contrast, with proper quadrature tuning voltage $V_{Q}$, quadrature is compensated and perfect mode-matching is achieved.

\section{FABRICATION}

Figure 4 shows the fabrication process flow of the anisotropic-wet-etched gyroscope. During the anisotropic wet etching, convex corners need to be protected. A two-mask LOCOS method [9] is used with modifications made to eliminate effects of misalignment. In the self-aligned process, the entire gyroscope pattern is defined on a first thick nitride mask layer. A second thin nitride layer partially covers the pattern and avoids the exposure of convex corners in the first wet etching. The thin nitride mask has irregular-shape openings. Wet etching undercuts the thin nitride and forms trenches aligned to the thick nitride mask. Therefore, small misalignment between the two nitride masks will not affect the final device geometry. Following Ref. [9], local oxidation and a second wet-etching step is performed after the first wet-etching, 
forming the final SCS structures with intact convex corners. After the device geometry is defined, polysilicon and sacrificial oxide surface micro-machining steps [10] are used to form horizontal and slanted electrodes with sub-micro gaps.

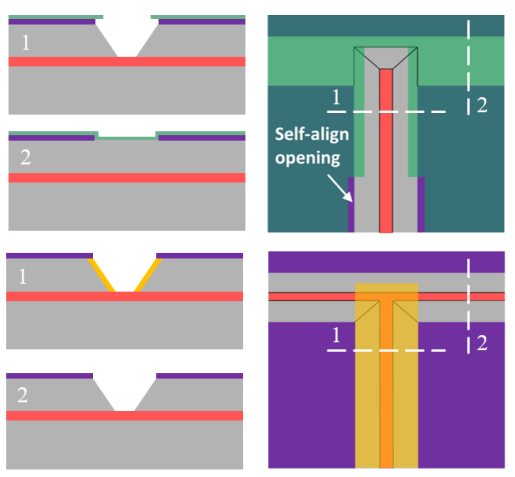

(a) Deposit and pattern thick LPCVD nitride layer to define trench shapes; deposit and pattern thin LPCVD nitride to form self-aligned openings; first $\mathrm{KOH}$ wet-etching.

b) Wet oxidation of SCS to form thick thermal oxide for (111) sidewall protection; blank etch nitride using RIE to remove thin nitride layer; second $\mathrm{KOH}$ wet-etching.

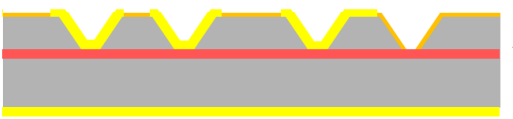

(c) Kemove nitride and oxide with RIE; deposit and pattern TEOS; wet oxidation to form sacrificial thin oxide.

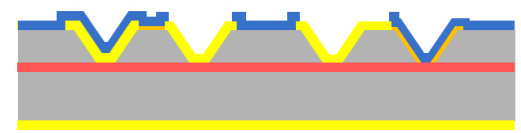

(d) Pattern thin oxide; deposit and pattern LPCVD polysilicon.

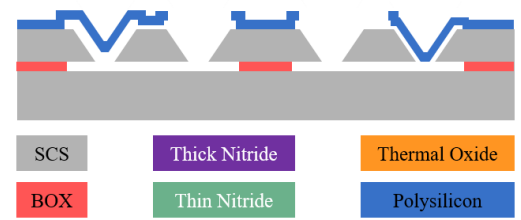

(e) Release device in $\mathrm{HF}$ solution and dry with super critical dryer.

Figure 4: Fabrication process flow for anisotropic-wet-etched pitch or roll gyroscope.

Prototypes of the gyroscope are fabricated on a $40 \mu \mathrm{m}$ (100) SOI wafer. $\mathrm{KOH}$ is chosen as the etchant for its high crystalline selectivity. Gap sizes of $\sim 500 \mathrm{~nm}$ are defined with sacrificial thermal oxide for top surface and slanted electrodes. Figure 5-7 show the SEM pictures of a fabricated gyroscope. The selfconfined nature of anisotropic wet etching results in very smooth slanted sidewalls. Convex corners are mostly protected although small imperfections are found due to excessive $\mathrm{KOH}$ etching (Fig. 6). Sub-micron transduction gaps are successfully formed both on top and on slanted sidewalls of the device with polysilicon electrodes stiff enough for designed operation voltages (Fig. 7).

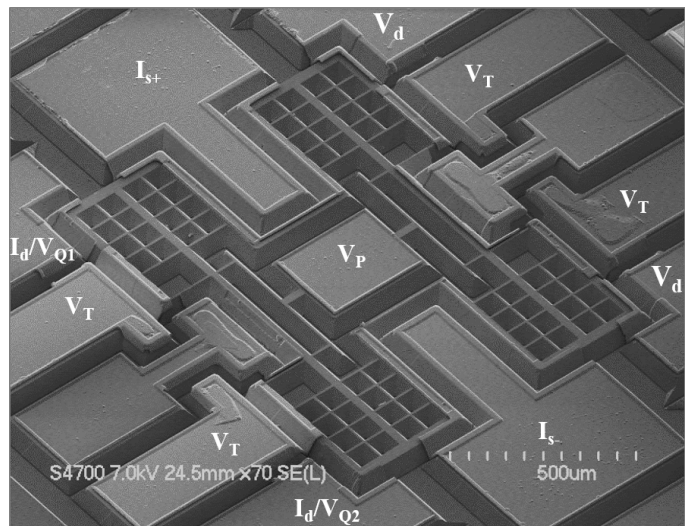

Figure 5: SEM picture of the wet-etched gyroscope. Electrical connections are labeled on the polysilicon pads, indicating the electrode configuration used in experimental measurements.

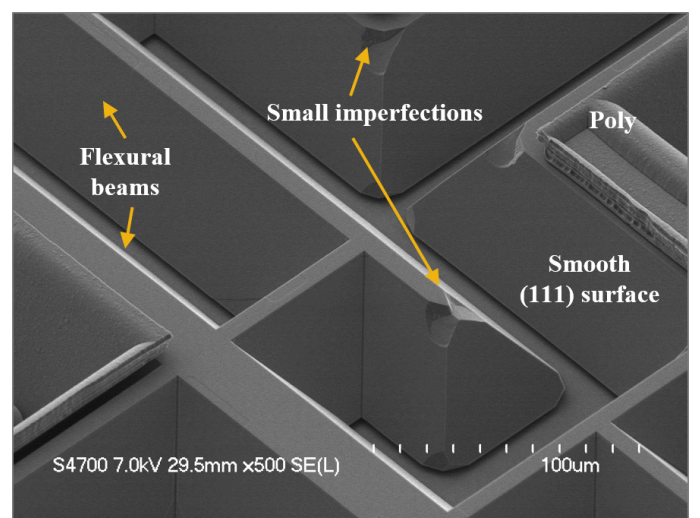

Figure 6: Close-up SEM picture of convex corner structure and wet-etched surface finish.

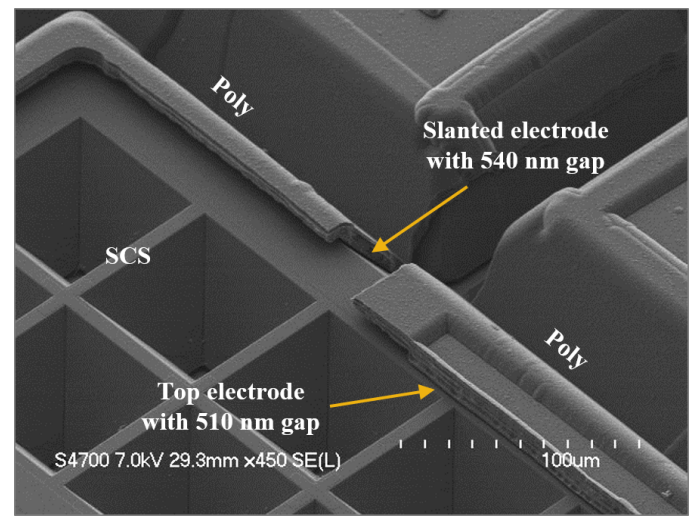

Figure 7: Close-up SEM picture of electrodes and sub-micron capacitive gaps on top and slanted sidewall of the gyroscope.

\section{MEASUREMENTS}

The quadrature tuning and mode-matching of fabricated gyroscope is characterized using a 4-port Agilent network analyzer with $V_{P}=23.35 \mathrm{~V}$. Drive and sense modes are actuated using separate channels, and the frequency response of each channel is recorded. Figure 8 shows the stiffness tuning behavior of the gyroscope without quadrature cancellation. As expected, veering phenomenon is observed where the eigen-modes push each other away when getting close and switch place without crossing at a common frequency. A minimum frequency-split of $\sim 60 \mathrm{~Hz}$ is found by tuning down sense mode frequency with $V_{T}$.

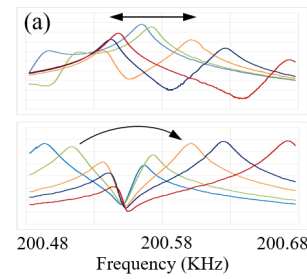

Drive mode frequency varies when sense mode passes by with changing tuning voltage

Sense mode switches place with drive mode without crossing at same frequency $-\mathrm{V}_{\mathrm{T}}=0 \mathrm{~V}-\mathrm{V}_{\mathrm{T}}=0.2 \mathrm{~V}-\mathrm{V}_{\mathrm{T}}=0.5 \mathrm{~V}$

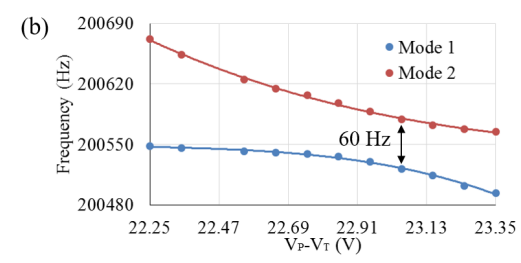

Figure 8: Measured sense mode frequency tuning behavior without quadrature tuning, showing frequency veering. (a) Frequency spectrum of drive and sense channels. (b) Peak frequencies. 


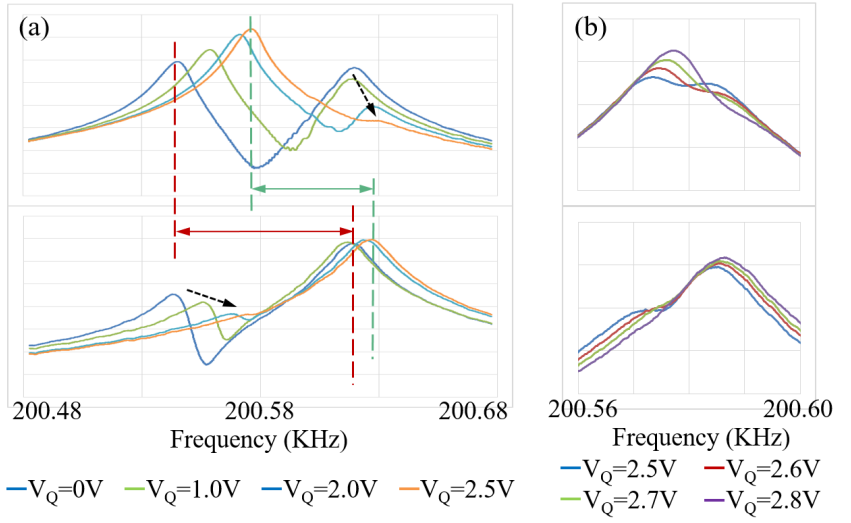

Figure 9: Measured quadrature tuning behavior of the gyroscope. (a) Coarse quadrature tuning with the presence of large spring mismatch. (b) Fine quadrature tuning after $V_{T}$ is adjusted to reduce spring mismatch.

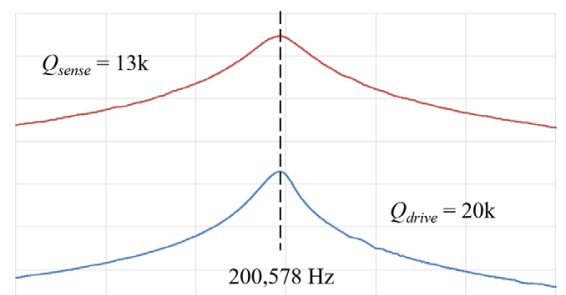

Figure 10: Mode-matched drive (blue) and sense (red) resonance peaks at 200,578 $\mathrm{Hz}$.
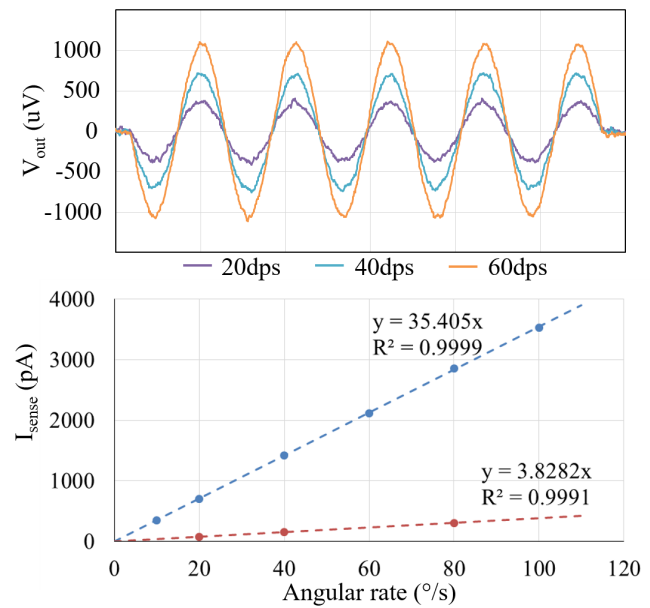

Figure 11: Measured rate response with mode-matched condition and scale factor comparison between mode-matched and modesplit conditions. TIA with $510 \mathrm{k} \Omega$ resistor is used for sense output.

Figure 9 shows the quadrature tuning effect using the slanted electrode. When proper quadrature tuning voltage is applied, the cross-coupling peak drops and the frequency-split reduces. Perfect mode-matching is achieved at $200 \mathrm{kHz}$ by fine tuning $V_{T}$ and $V_{Q}$, showing quality factors of $20 \mathrm{k}$ and $13 \mathrm{k}$ for drive and sense modes, respectively (Fig. 10).

Angular rate response of the gyroscope is also characterized (Fig. 11). The mode-matched scale factor of the gyroscope is measured to be $35.4 \mathrm{pA} / \% / \mathrm{s}$. For comparison, sensitivity is also measured with a minimum frequency-split without quadrature nulling, which gives a scale factor of $3.8 \mathrm{pA} / \% / \mathrm{s}$. Table 1 lists the measurement results for the anisotropic-wet-etched gyroscope.
Table 1: Measured performance parameters.

\begin{tabular}{|l|c|}
\hline Mode-matched frequency & $200.578 \mathrm{kHz}$ \\
\hline Mode-matched $Q$ & $Q_{d} \sim 20 \mathrm{k}, Q_{s} \sim 13 \mathrm{k}$ \\
\hline$\Delta f_{\min }$ without $V_{Q}$ & $\sim 60 \mathrm{~Hz}$ \\
\hline$\Delta f_{\min }$ with $V_{Q}$ & $0 \mathrm{~Hz}$ \\
\hline Scale factor $\left(\right.$ w/o $V_{Q}$ ) & $3.8 \mathrm{pA} / \% / \mathrm{s}$ \\
\hline Scale factor $\left(\right.$ with $\left.V_{Q}\right)$ & $35.4 \mathrm{pA} / \% / \mathrm{s}$ \\
\hline
\end{tabular}

\section{CONCLUSIONS}

Quadrature error significantly limits the performance of outof-plane pitch and roll MEMS gyroscopes. Through the use of slanted electrodes enabled by anisotropic wet etching of SCS, we successfully demonstrate electrostatic quadrature cancellation in out-of-plane resonant gyroscopes. The elimination of quadrature allows perfect mode-matching of the gyroscope, resulting in $\sim 10 \times$ improvement in sensitivity. In addition, the anisotropic-wet-etched design reduces the thickness dependency of frequency-split and eliminates expansive DRIE steps, showing promises in improving the yield and fabrication cost of pitch and roll resonant gyroscopes.

\section{ACKNOWLEDGEMENT}

This work is supported by the DARPA MTO, Single-Chip Timing and Inertial Measurement Unit (TIMU) program under contract \# N66001-11-C-4176. The authors want thank D. Serrano for discussions on gyroscopes, and A. Daruwalla and cleanroom staff at Georgia Tech for fabrication assistance.

\section{REFERENCES}

[1] F. Ayazi, "Multi-DOF Inertial MEMS: From Gaming to Dead-Reckoning," in Tech. Dig. of the 16th International Conference on Solid-State Sensors, Actuators and Microsystems, Beijing, China, (2011), pp. 2805-2808.

[2] L. Ojeda, and J. Borenstein, "Personal Dead-reckoning System for GPS denied Environments," in Safety, Security and Rescue Robotics, IEEE International Workshop on, (2007), pp.1-6, 27-29.

[3] C. Randell, et al., "Personal position measurement using dead reckoning," in Proc. of the 7th International Symposium on Wearable Computers, (2003), pp. 166-173.

[4] M. F. Zaman, et al., "A Mode-Matched Silicon-Yaw TuningFork Gyroscope with Subdegree-Per-Hour Allan Deviation Bias Instability," IEEE J. Microelectromech, Syst., 17, 6, (2008), pp. 1526-1536.

[5] A. A. Trusov, et al., "Ultra-High Q Silicon Gyroscopes with Interchangeable Rate and Whole Angle Modes of Operation," Proc. IEEE Sensors, (2010), pp. 864-867.

[6] H. Johari and F. Ayazi, "Capacitive Bulk Acoustic Wave Silicon Disk Gyroscopes," Tech. Dig. IEEE Int. Electron Devices Meeting, San Francisco, CA, (2006), pp. 513-516.

[7] S. Vidoli, et al., "Veering phenomena in systems with gyroscopic coupling," J. App. Mech., 72, (2005), pp. 641-647.

[8] B. J. Gallacher, et al., "Electrostatic correction of structural imperfections present in a microring gyroscope," J. Microelectromech. Syst., 14, 2, (2005), pp. 221-234.

[9] P. Pal and S. Chandra, "A novel process for perfect convex corner realization in bulk micromachining," J. Micromech. Microeng. 14, (2004), pp. 1416-1420.

[10] F. Ayazi and K. Najafi, "High Aspect-Ratio Combined Poly and Single-Crystal Silicon (HARPSS) MEMS Technology," IEEE J. Microelectromech. Syst., 9, (2000), pp. 288-294.

\section{CONTACT}

*H. Wen, tel: +1-404-317-7766; haoran.wen@gatech.edu 